\title{
USO Y PERCEPCIONES DE LAS TECNOLOGÍAS DE INFORMACIÓN Y COMUNICACIÓN EN PACIENTES CON HIPERTENSIÓN ARTERIAL, DISLIPIDEMIA O DIABETES DE UN HOSPITAL NACIONAL DE LIMA, PERÚ
}

\author{
Andrea C. Rivas-Nieto ${ }^{1,2, a}$, Germán Málagaa,3,b, Paulo Ruiz-Grosso ${ }^{1,3,4, c}$, Carlos A. Huayanay-Espinoza ${ }^{1, d}$, \\ Walter H. Curioso ${ }^{5, e}$
}

\begin{abstract}
RESUMEN
El presente estudio tuvo como objetivo determinar el uso y percepciones hacia las tecnologías de información y comunicación (TIC), en 206 pacientes portadores de hipertensión arterial, dislipidemia y diabetes, reclutados de la consulta externa en un hospital nacional de Lima, Perú. El $54,4 \%$ fueron adultos mayores y $70,4 \%$ mujeres. El uso diario de llamadas por celular fue $44,7 \%$; la mayoría nunca había usado una computadora $(78,2 \%)$, correo electrónico (84\%) o Internet $(84 \%)$. Muchos nunca han enviado $(80,6 \%)$ o recibido $(69,9 \%)$ un mensaje de texto. El $70 \%$ ha olvidado alguna vez tomar su medicina. Al 72,8\% le gustaría que le recuerden tomar sus medicinas y $67,9 \%$ tiene algún familiar que podría ayudarlos a acceder a las TIC. Pese al bajo uso de las TIC en esta población, existe predisposición y expectativa por los pacientes a participar en programas que las implementen.
\end{abstract}

Palabras clave: Tecnología de la información; Hipertensión; Dislipidemias; Diabetes mellitus (fuente: DeCS BIREME).

\section{USE AND PERCEPTIONS OF INFORMATION AND COMMUNICATION TECHNOLOGIES IN PATIENTS WITH HYPERTENSION, DYSLIPIDEMIA OR DIABETES IN A NATIONAL HOSPITAL IN LIMA, PERU}

\begin{abstract}
This study aimed to determine the use and perceptions towards information and communication technologies (ICT) in 206 patients with arterial hypertension, dyslipidemia and diabetes, recruited from the outpatient clinic in a national hospital in Lima, Peru. $54.4 \%$ were older adults and $70.4 \%$ were women. The use of daily phone calls was $44.7 \%$. Most had never used a computer $(78.2 \%)$, email $(84 \%)$ or the Internet $(84 \%)$. Many have never sent $(80.6 \%)$ or received $(69.9 \%)$ a text message. $70 \%$ had at some time forgotten to take their medicine. $72.8 \%$ would like to be reminded to take their medication and $67.9 \%$ had a family member who could help them with access to ICT. Despite the low use of ICT in this population, there is willingness and expectation from the patients to participate in programs that implement them.
\end{abstract}

Key words: Information technology; Hypertension; Dyslipidemias; Diabetes mellitus (source: MeSH NLM).

\section{INTRODUCCIÓN}

El aumento de la carga de enfermedad de las enfermedades crónicas en países de medianos y bajos ingresos y el alto costo que representan, despierta el interés por desarrollar nuevas estrategias de prevención y seguimiento de pacientes ${ }^{(1)}$. De allí surge la necesidad de desarrollar programas e intervenciones innovadoras en salud que involucren tecnologías móviles e Internet, debido principalmente a la efectividad para la recolección de datos y para el envío de información, así como la mejor calidad de esta ${ }^{(2)}$.

\footnotetext{
Facultad de Medicina, Universidad Peruana Cayetano Heredia. Lima, Perú.

Sociedad Científica de Estudiantes de Medicina Cayetano Heredia. Lima, Perú.

Hospital Nacional Cayetano Heredia. Lima, Perú.

Grupo de Trabajo en Salud Mental, Universidad Peruana Cayetano Heredia. Lima, Perú.

Consejo Nacional de Ciencia, Tecnología e Innovación Tecnológica (CONCYTEC). Lima, Perú.

Estudiante de medicina; ${ }^{\mathrm{b}}$ médico internista; ${ }^{\mathrm{c}}$ médico residente de Psiquiatría; ${ }^{\mathrm{d}}$ tecnólogo médico; ${ }^{\mathrm{e}}$ médico, maestro en Salud Pública, doctor en Informática Biomédica

Recibido: : 17-03-15 Aprobado: 27-05-15
}

Citar como: Rivas-Nieto AC, Málaga G, Ruiz-Grosso P, Huayanay-Espinoza CA, Curioso WH. Uso y percepciones de las tecnologías de información y comunicación en pacientes con hipertensión arterial, dislipidemia o diabetes de un hospital nacional de Lima, Perú. Rev Peru Med Exp Salud Publica. 2015;32(2):283-8. 
Intervenciones similares se han desarrollado con éxito en países desarrollados, facilitado por el hecho que la población tiene mayor acceso a dispositivos electrónicos con múltiples funciones ${ }^{(3,4)}$. Entre algunos programas que usan estas tecnologías, se puede mencionar el monitoreo de glucosa en pacientes con diabetes tipo I y II, sistemas de apoyo para dejar de fumar, seguimiento de pacientes con asma y enfermedades autoinmunes ${ }^{(2-5)}$, entre otras.

En países de medianos y bajos ingresos, las intervenciones realizadas haciendo uso de tecnología móvil e Internet, sobre todo en el campo de las enfermedades infecciosas, han demostrado ser exitosas, aun con presupuestos limitados. Algunas de las áreas en las que han sido aplicadas son: sistemas de salud, vigilancia epidemiológica, educación a la población y monitoreo de estrategias de salud ${ }^{(6)}$. Además, en el área clínica, las tecnologías móviles posibilitan un diagnóstico temprano, lo que resulta en un mejor control de estas enfermedades. Esto incluye seguimiento de pacientes y mejoramiento de la adherencia al tratamiento (7), lo que constituye un importante objetivo para los programas de salud pública, ya que hasta $60 \%$ de los pacientes no toman sus medicinas como son prescritas, por lo que todavía hay mucho por explorar y mejorar ${ }^{(8)}$.

La información sobre adherencia al tratamiento en Perú, es aún limitada. En un estudio realizado en un hospital de Lima por Curioso et al. con pacientes con diabetes tipo II, se observó que $43,3 \%$ de las personas encuestadas desearían participar en un programa usando celulares e Internet ya que olvidan tomar sus medicinas y un $39 \%$ desearían tener recordatorios de su tratamiento ${ }^{(6)}$.

Los objetivos de este estudio son describir la proporción y frecuencia de uso de TIC en pacientes con dislipidemia (DLP), diabetes (DM) e hipertensión arterial (HTA), explorar el porcentaje de adherencia al tratamiento y la percepción de participar en intervenciones utilizando tecnologías de información y comunicación (TIC) en esta población.

\section{EL ESTUDIO}

Se realizó un estudio transversal descriptivo en 206 pacientes mayores de 18 años con al menos uno de los diagnósticos considerados (diabetes, hipertensión o dislipidemia), que se encontraban en la sala de espera de los consultorios de cardiología, endocrinología y medicina interna; quienes asintieron a responder la encuesta. Se entregó una hoja informativa con la naturaleza del estudio.
Se empleó una encuesta validada por expertos, utilizada previamente por Curioso et al. en 2009 para DM y se realizaron pequeñas modificaciones para diferenciar los tres diagnósticos (HTA, DM, DLP) ${ }^{(6)}$, tomando en cuenta que algunos pacientes presentaban dos o más condiciones crónicas.

Mediante dicho cuestionario se recolectó los datos demográficos de los pacientes (sexo, edad, lugar de nacimiento, procedencia, grado de instrucción y estado civil), el uso de tecnologías de la información (celulares e Internet), la información sobre su enfermedad entregada por el hospital, así como el tipo y los medios por los cuales deseaban obtener mayor información. Se evaluó la adherencia a los servicios médicos y al tratamiento, cuantificando la frecuencia de visitas al hospital, el número de medicamentos que usan, la frecuencia con la que se los habían prescrito y las veces que olvidaron tomarlos. Se utilizó una escala visual análoga del 1 al 100, para que los pacientes autocalifiquen su cumplimiento de toma de medicamentos. Finalmente, los pacientes respondieron Sí/NO a su deseo de participar de una intervención usando celulares o Internet, a manera de evaluar la factibilidad de una intervención.

El análisis se realizó mediante el software Stata 12 (StataCorp, College Station, TX, USA), y estuvo basado en un análisis descriptivo y univariado.

El protocolo del estudio fue aprobado por la Facultad de Medicina Alberto Hurtado y el Comité de Ética de la Universidad Peruana Cayetano Heredia, así como al Comité Institucional de Ética del Hospital Nacional Cayetano Heredia.

\section{HALLAZGOS}

La edad media de los 206 pacientes entrevistados, fue $60,5 \pm 13,2$ años; $70,4 \%$ (145) de sexo femenino, la mayoría con estado civil viudo $48,1 \%$ (99) y $76,2 \%$ (157) con educación escolar, proveniente en un 34\% (70) del distrito de San Martín de Porres, Lima; y 62,6\% (129) con lugar de nacimiento en provincia.

En la Tabla 1 se muestran los hallazgos del uso de tecnologías. Se observó que el uso diario de llamadas por celular fue de 44,7\% (92). Se observó, así mismo, que $78,2 \%$ (161) de los participantes nunca ha usado una computadora; $84 \%$ (173) no ha usado correo electrónico; $84 \%$ (173) nunca ha usado Internet; 80,6\% (166) nunca ha enviado un mensaje por celular, y $69,9 \%$ (144) nunca ha recibido un mensaje de texto. 
Tabla 1. Uso de tecnología de información y comunicación

\begin{tabular}{|c|c|c|}
\hline & $\mathrm{n}$ & $(\%)$ \\
\hline \multicolumn{3}{|l|}{ Uso de computadoras } \\
\hline Nunca & 161 & $(78,2)$ \\
\hline Al menos una vez/año & 2 & $(1,0)$ \\
\hline Al menos una vez/mes & 4 & $(1,9)$ \\
\hline Al menos una vez/semana & 23 & $(11,2)$ \\
\hline Todos los días & 16 & $(7,8)$ \\
\hline \multicolumn{3}{|l|}{ Uso de correo electrónico } \\
\hline Nunca & 173 & $(84,0)$ \\
\hline Al menos una vez/año & 2 & $(1,0)$ \\
\hline Al menos una vez/mes & 5 & $(2,4)$ \\
\hline Al menos una vez/semana & 16 & $(7,8)$ \\
\hline Todos los días & 10 & $(4,9)$ \\
\hline \multicolumn{3}{|l|}{ Navegar por Internet } \\
\hline Nunca & 173 & $(84,0)$ \\
\hline Al menos una vez/mes & 3 & $(1,5)$ \\
\hline Al menos una vez/semana & 19 & $(9,2)$ \\
\hline Todos los días & 11 & $(5,3)$ \\
\hline \multicolumn{3}{|l|}{ Uso de chat } \\
\hline Nunca & 188 & $(91,3)$ \\
\hline Al menos una vez/año & 1 & $(0,5)$ \\
\hline Al menos una vez/mes & 3 & $(1,5)$ \\
\hline Al menos una vez/semana & 9 & $(4,4)$ \\
\hline Todos los días & 5 & $(2,4)$ \\
\hline \multicolumn{3}{|c|}{ Búsqueda de información de salud en Internet } \\
\hline Nunca & 179 & $(87,0)$ \\
\hline Al menos una vez/año & 1 & $(0,5)$ \\
\hline Al menos una vez/mes & 7 & $(3,4)$ \\
\hline Al menos una vez/semana & 13 & $(6,3)$ \\
\hline Todos los días & 6 & $(2,9)$ \\
\hline \multicolumn{3}{|c|}{ Realiza o recibe llamadas por celular } \\
\hline Nunca & 73 & $(35,4)$ \\
\hline Al menos una vez/mes & 4 & $(1,9)$ \\
\hline Al menos una vez/semana & 37 & $(18,0)$ \\
\hline Todos los días & 92 & $(44,7)$ \\
\hline \multicolumn{3}{|c|}{ Envía mensaje de texto por celular } \\
\hline Nunca & 166 & $(80,6)$ \\
\hline Al menos una vez/año & 2 & $(1,0)$ \\
\hline Al menos una vez/mes & 3 & $(1,5)$ \\
\hline Al menos una vez/semana & 17 & $(8,3)$ \\
\hline Todos los días & 18 & $(8,7)$ \\
\hline \multicolumn{3}{|c|}{ Recibe mensaje de texto por celular } \\
\hline Nunca & 144 & $(69,9)$ \\
\hline Al menos una vez/mes & 6 & $(2,9)$ \\
\hline Al menos una vez/semana & 28 & $(13,6)$ \\
\hline Todos los días & 28 & $(13,6)$ \\
\hline
\end{tabular}

Sobre la información que reciben en el hospital, el $48,1 \%$ (99) de los pacientes consideran que reciben adecuada cantidad de información y el $51,1 \%$ (106) cree que es útil. Asimismo, 98,1\% de los entrevistados desearía recibir más información sobre su enfermedad, en especial en temas relacionados con manejo, cuidado y prevención $54,3 \%$ (102), sobre todo mediante charlas $52,1 \%$ (98), tal como se muestra en la Tabla 2.
Tabla 2. Información recibida sobre hipertensión arterial, diabetes y dislipidemia por los participantes

\begin{tabular}{|c|c|c|}
\hline & $\mathbf{n}$ & $(\%)$ \\
\hline \multicolumn{3}{|c|}{$\begin{array}{l}\text { Calificación de la cantidad de información recibida del } \\
\text { personal de salud }\end{array}$} \\
\hline Muy poca & 63 & $(30,6)$ \\
\hline Menos que adecuada & 25 & $(12,1)$ \\
\hline Adecuada & 99 & $(48,1)$ \\
\hline Más de lo adecuado & 17 & $(8,3)$ \\
\hline Demasiada información & 1 & $(0,5)$ \\
\hline No sabe o no responde & 1 & $(0,5)$ \\
\hline \multicolumn{3}{|l|}{ Utilidad de la información recibida } \\
\hline Nada útil & 5 & $(2,4)$ \\
\hline Poco útil & 22 & $(10,7)$ \\
\hline Medianamente útil & 26 & $(12,6)$ \\
\hline Útil & 106 & $(51,5)$ \\
\hline Muy útil & 39 & $(18,9)$ \\
\hline No sabe o no responde & 8 & $(3,9)$ \\
\hline \multicolumn{3}{|c|}{$\begin{array}{l}\text { Desea recibir información sobre el cuidado de su } \\
\text { enfermedad }\end{array}$} \\
\hline 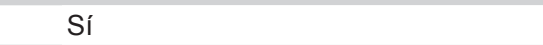 & 188 & $(91,3)$ \\
\hline No & 16 & $(7,8)$ \\
\hline No sabe o no responde & 2 & $(1,0)$ \\
\hline \multicolumn{3}{|l|}{ Tipo de información que desea recibir* } \\
\hline Manejo, cuidado y prevención & 102 & $(54,3)$ \\
\hline Alimentación y estilo de vida & 39 & $(20,7)$ \\
\hline Información general de la enfermedad & 19 & $(10,1)$ \\
\hline Complicaciones y riesgo & 13 & $(6,9)$ \\
\hline Información sobre trasplante & 2 & $(1,1)$ \\
\hline No sabe o no responde & 13 & $(6,9)$ \\
\hline \multicolumn{3}{|l|}{$\begin{array}{l}\text { Preferencia de medios para recibir } \\
\text { información (opción múltiple) }{ }^{*}\end{array}$} \\
\hline Charlas & 98 & $(52,1)$ \\
\hline Papel (folletos, trípticos, afiches) & 35 & $(18,6)$ \\
\hline Correos electrónicos & 7 & $(3,7)$ \\
\hline Internet (página web) & 3 & $(1,6)$ \\
\hline Directamente con el médico & 5 & $(2,7)$ \\
\hline Teléfono fijo & 2 & $(1,1)$ \\
\hline Más de un medio de información & 38 & $(20,2)$ \\
\hline \multicolumn{3}{|l|}{ Persona que lo ayudaría a usar Internet** } \\
\hline Hijo(a) & 103 & $(73,6)$ \\
\hline Nieto(a) & 15 & $(10,7)$ \\
\hline Sobrino(a) & 11 & $(7,9)$ \\
\hline Hermano(a) menor & 5 & $(3,6)$ \\
\hline Otros & 6 & $(4,3)$ \\
\hline
\end{tabular}

*Total de encuestados $=188,{ }^{* *}$ Total de encuestados $=140$

La adherencia a los servicios y tratamiento se detalla en la Tabla 3. Se observa que el $61 \%$ (126) visita al menos una vez al mes el hospital y el $70 \%$ (119) de los pacientes entrevistados ha olvidado alguna vez tomar su medicina y solo $31,6 \%$ (54) considera que cumple $\geq 95 \%$ la toma de sus medicinas. La causa más frecuente de la omisión de tratamiento fue el olvido, expresado por el 70,3\% (83) de los sujetos. 
Tabla 3. Adherencia a los servicios médicos y al tratamiento

\begin{tabular}{|c|c|c|}
\hline & $\mathbf{n}$ & $(\%)$ \\
\hline \multicolumn{3}{|c|}{ Frecuencia de visita a establecimiento de salud } \\
\hline Al menos una vez al año & 52 & $(25,2)$ \\
\hline Al menos una vez al mes & 126 & $(61,2)$ \\
\hline Al menos una vez a la semana & 16 & $(7,8)$ \\
\hline No sabe o no responde & & $(5,8)$ \\
\hline \multicolumn{3}{|c|}{$\begin{array}{l}\text { Calificación de calidad de atención del establecimiento } \\
\text { de salud }\end{array}$} \\
\hline Muy mala & 2 & $(1,0)$ \\
\hline Mal & 21 & $(10,2)$ \\
\hline Buena & 141 & $(68,5)$ \\
\hline Muy buena & 27 & $(13,1)$ \\
\hline Excelente & 8 & $(3,9)$ \\
\hline No sabe o no responde & 7 & $(3,4)$ \\
\hline \multicolumn{3}{|l|}{ Tratamiento actual } \\
\hline Solo HTA & 80 & $(38,8)$ \\
\hline Solo diabetes & 30 & $(15,6)$ \\
\hline Solo dislipidemia & 10 & $(4,9)$ \\
\hline HTA y diabetes & 21 & $(10,2)$ \\
\hline HTA y dislipidemia & 12 & $(5,8)$ \\
\hline Diabetes y dislipidemia & 5 & $(2,4)$ \\
\hline HTA, diabetes y dislipidemia & 12 & $(5,8)$ \\
\hline Sin tratamiento & 36 & $(17,5)$ \\
\hline \multicolumn{3}{|c|}{ Alguna vez olvidó tomar/inyectar su medicina $(n=170)$} \\
\hline Sí & 119 & $(70,0)$ \\
\hline No & 51 & $(30,0)$ \\
\hline \multicolumn{3}{|c|}{$\begin{array}{l}\text { Porcentaje de cumplimiento de las indicaciones en el } \\
\text { tratamiento del médico los últimos } 30 \text { días }\end{array}$} \\
\hline Menos de 50 & 16 & $(9,4)$ \\
\hline 51 a 65 & 9 & $(5,3)$ \\
\hline 66 a 80 & 29 & $(17,0)$ \\
\hline 81 a 95 & 63 & $(36,8)$ \\
\hline Más de 95 & 54 & $(31,6)$ \\
\hline \multicolumn{3}{|c|}{$\begin{array}{l}\text { Motivos por los cuales no tomó su medicina/inyección en } \\
\text { los últimos } 30 \text { días ( } n=118)\end{array}$} \\
\hline Olvido & 83 & $(70,3)$ \\
\hline Trabajo y/o ocupación & 13 & $(11,0)$ \\
\hline Se quedó dormido & 3 & $(2,5)$ \\
\hline Problemas y preocupaciones & 6 & $(5,1)$ \\
\hline Otros & 10 & $(8,5)$ \\
\hline \multicolumn{3}{|c|}{ Le gustaría que le recuerden tomar/inyectar sus medicinas } \\
\hline Sí & 99 & $(72,8)$ \\
\hline No & 37 & $(27,2)$ \\
\hline
\end{tabular}

HTA: hipertensión arterial

Ante la factibilidad de una intervención, al 72,8\% (99) le gustaría que le recuerden tomar sus medicinas y $67,9 \%$ (140) de los pacientes tienen algún familiar que podría ayudarlos a acceder a las tecnologías (Tabla 4).

\section{DISCUSIÓN}

En este estudio se encontró que los pacientes con enfermedades crónicas entrevistados son predominantemente adultos mayores con bajo nivel de uso de tecnologías de información y comunicación (TIC).
Tabla 4. Factibilidad de una intervención usando celulares o internet

\begin{tabular}{|c|c|c|}
\hline & $\mathbf{n}$ & $(\%)$ \\
\hline \multicolumn{3}{|c|}{$\begin{array}{l}\text { Participación en algún programa o taller } \\
\text { para mejorar su tratamiento para HTA, } \\
\text { diabetes o dislipidemia }\end{array}$} \\
\hline Sí & 14 & $(6,8)$ \\
\hline No & 184 & $(89,3)$ \\
\hline No sabe o no responde & 8 & $(3,9)$ \\
\hline \multicolumn{3}{|c|}{$\begin{array}{l}\text { Utilidad de participación en ese programa } \\
\text { o taller }\end{array}$} \\
\hline Medianamente útil & 3 & $(21,4)$ \\
\hline Útil & 4 & $(28,6)$ \\
\hline Muy útil & 5 & $(35,7)$ \\
\hline No sabe o no responde & 2 & $(14,3)$ \\
\hline \multicolumn{3}{|c|}{$\begin{array}{l}\text { Interés en participar en un programa } \\
\text { usando celulares o Internet para } \\
\text { recordarles la toma de pastillas }\end{array}$} \\
\hline Sí & 113 & $(54,9)$ \\
\hline No & 91 & $(44,2)$ \\
\hline No sabe o no responde & 2 & $(1,0)$ \\
\hline \multicolumn{3}{|c|}{$\begin{array}{l}\text { Interés en participar en un programa } \\
\text { usando celulares o Internet para el } \\
\text { seguimiento de su HTA, dislipidemia o } \\
\text { diabetes }\end{array}$} \\
\hline Sí & 122 & $(59,2)$ \\
\hline No & 82 & $(39,8)$ \\
\hline No sabe o no responde & 2 & $(1,0)$ \\
\hline
\end{tabular}

HTA: hipertensión arterial

A diferencia de estudios previos ${ }^{(6)}$, se observó un incremento en el uso de telefonía celular para hacer llamadas $(30,5$ a $44,7 \%)$, hallazgo que se explica, posiblemente, por la rápida expansión de la telefonía celular en el país. Asimismo, la gran mayoría de pacientes entrevistados desea recibir más información sobre su enfermedad, de preferencia mediante charlas, especialmente sobre manejo, cuidado y prevención (54,3\%). Esto concuerda con el estudio de Curioso et al., donde se encontró que la mayoría de participantes prefería recibir información mediante charlas o medios escritos.

Pese al bajo uso de TIC, más de la mitad de los pacientes están dispuestos a recibir recordatorios $(54,9 \%)$ y a ser parte de programas de seguimiento $(59,2 \%)$ usando celulares e Internet. Esto último ofrece una perspectiva positiva sobre el uso de las TIC, y debe ser abordado en futuros estudios de intervención.

Entre los hallazgos de este estudio también resalta la baja adherencia de los pacientes a su tratamiento, tomando 
en cuenta que $70,3 \%$ olvida tomar sus medicinas, y solo una tercera parte $(31 \%)$ considera que cumple adecuadamente lo prescrito, pese a acudir de forma frecuente al hospital (61,2\% una vez al mes). Porcentajes similares han sido hallados en otros estudios, donde la adherencia al tratamiento en hipertensión oscila entre 37 y $52 \%{ }^{(9)}$, asimismo, el porcentaje de pacientes con pobre control glicémico llega a un $73,4 \%$ pese a estar medicados ${ }^{(10)}$. Estos datos son de gran importancia en salud pública, ya que demuestran que se debe aunar esfuerzos en promover la adherencia con estrategias innovadoras y amigables para las personas ${ }^{(11)}$.

Un aspecto a destacar es que la población estudiada tiene predisposición y expectativas por participar en programas: por ejemplo, $72,8 \%$ desean que se les recuerde la toma de las medicinas. Otro interesante hallazgo es que los pacientes tienen familiares que podrían ayudarlos a usar las TIC. El estudio previo de Curioso et al. mostró similares hallazgos ${ }^{(6)}$. Estudios publicados en países desarrollados respaldan la disposición de los cuidadores de participar en programas usando TIC, existe la posibilidad de explorar el rol de ellos en el mejoramiento de la adherencia de los pacientes y la calidad de vida ${ }^{(12-14)}$.

Entre las intervenciones planteadas para el futuro, se puede considerar el envío de recordatorios de la toma de medicamentos para mejorar la adherencia, mensajes cortos informativos sobre las enfermedades y sus cuidados, además de crear un sistema de monitoreo continuo de valores de glucosa, presión arterial, entre otros, mediante el envío de información por celulares o Internet, mejorando de esta manera la detección previa de complicaciones. Varios programas con este tipo de tecnologías han tenido éxito según los estudios realizados en diferentes partes del mundo $(5,7,15,16)$. Un estudio reciente muestra el esfuerzo por generar mensajes de texto comprensibles para personas de países latinoamericanos, incluyendo al Perú, lo cual indica que se está trabajando en el desarrollo de métodos de comunicación mediante TIC y acerca la posibilidad de usar recordatorios similares en el futuro adaptados a la realidad peruana ${ }^{(17)}$.
Entre las limitaciones del estudio se puede mencionar que la muestra no fue aleatoria, por no contar con una lista definida de pacientes, especialmente de aquellos que acudían por primera vez a consulta. Sin embargo, el estudio presenta fortalezas pues se realizó en un hospital que es centro de referencia a nivel nacional, lo cual permitió contar con una muestra diversa de pacientes. Así mismo, se empleó una encuesta validada y previamente utilizada en un hospital peruano.

En conclusión, aunque este estudio muestra un bajo uso de las TIC, se observa un incremento en el uso de telefonía celular. Se necesita investigar nuevas estrategias de comunicación con los pacientes, para mejorar la baja adherencia detectada al tratamiento prescrito, lo que ocurre, pese a acudir con regularidad a sus citas. Los mensajes de texto e Internet pueden ser útiles para el desarrollo de programas de salud, al contar con la aceptación de pacientes y cuidadores, quienes podrían ser agentes clave en el desarrollo e implementación de estos programas. Finalmente, se deben realizar nuevos estudios que evalúen el uso de las tecnologías de comunicación, que constituye un área en permanente evolución.

Agradecimientos: en memoria del Dr. Francisco Tejada Reátegui: agradecemos su apoyo incondicional a este proyecto y al desarrollo de la investigación en nuestro país.

Contribuciones de autoría: ACRN, WHC y GMR participaron en la redacción del protocolo de investigación, ejecución, análisis y redacción del manuscrito; PRG participó en la elaboración del protocolo, análisis estadístico y redacción del manuscrito. CHE contribuyó con el análisis estadístico y redacción del manuscrito. Todos los autores aprobaron la versión final a publicar.

Fuentes de financiamiento: este estudio fue ganador de la Beca Anual de Medicina "Francisco Tejada y Semíramis Reátegui" de la Universidad Peruana Cayetano Heredia.

Conflictos de interés: Los autores declaran no tener conflictos de interés.

\section{REFERENCIAS BIBLIOGRÁFICAS}

1. Abegunde DO, Mathers CD, Adam T, Ortegon M, Strong K. The burden and costs of chronic diseases in low-income and middleincome countries Lancet. 2007 Dec 8;370(9603):1929-38.

2. Anta R, El-Wahab S, Giuffrida A. Salud Móvil: El potencial de la telefonía celular para llevar la salud a la mayoría. Brasilia: Banco Interamericano de Desarrollo; 2009.

3. The Earth Institute, Columbia University. Barriers and Gaps Affecting mHealth in Low and Middle Income Countries. Washington DC: mHealth Alliance; 2010.
4. Wei J, Hollin I, Kachnowski S. A review of the use of mobile phone text messaging in clinical and healthy behaviour interventions. J Telemed Telecare. 2011;17(1):41-8. doi: $10.1258 /$ jtt.2010.100322.

5. Free C, Phillips G, Felix L, Galli L, Patel V, Edwards P. The effectiveness of M-health 
technologies for improving health and health services: a systematic review protocol. BMC Res Notes. 2010 Oct 6;3:250. doi: 10.1186/1756-0500-3-250.

6. Curioso WH, Gozzer E, Valderrama M, Rodriguez-Abad J, Villena J, Villena A. Uso y percepciones hacia las tecnologías de información y comunicación en pacientes con diabetes, en un hospital público del Perú. Rev Peru Med Exp Salud Publica. 2009;26(2):161-7.

7. Curioso WH. Salud móvil en atención primaria. En: Carnicero J, Fernández A, Rojas D; Naciones Unidas. Manual de salud electrónica para directivos de servicios y sistemas de salud. Aplicaciones de las TIC a la atencion primaria de salud. Santiago de Chile: Naciones Unidas; 2014. p. 299-314.

8. Dunbar-Jacob J, Mortimer-Stephens MK. Treatment adherence in chronic disease. J Clin Epidemiol. 2001 Dec;54 Suppl 1:S57-60.

9. Carhuallanqui R, Diestra-Cabrera G, Tang-Herrera J, Málaga G. Adherencia al tratamiento farmacológico en pacientes hipertensos atendidos en un hospital general. Rev Med Hered. 2010;21(4):197-201.

10. Ramos W, López T, Revilla L, More L, Huamaní M, Pozo M. Resultados de la vigilancia epidemiológica de diabetes mellitus en hospitales notificantes del Perú, 2012. Rev Peru Med Exp Salud Publica. 2014;31(1):9-15.

11. Hall AK, Cole-Lewis H, Bernhardt JM. Mobile text messaging for health: a systematic review of reviews. Annu Rev Public Health. 2015 Mar 18;36:393-415. doi: 10.1146/annurevpublhealth-031914-122855.

12. Czaja SJ, Rubert MP. Telecommunications technology as an aid to family caregivers of persons with dementia. Psychosom Med. 2002 MayJun;64(3):469-76.

13. Marziali E, Donahue P. Caring for others: Internet video-conferencing group intervention for family caregivers of older adults with neurodegenerative disease. Gerontologist. 2006 Jun;46(3):398-403.

14. Gustafson DH Sr, McTavish F, Gustafson DH Jr, Mahoney JE, Johnson RA, Lee JD, et al. The effect of an information and communication technology (ICT) on older adults' quality of life: study protocol for a randomized control trial. Trials. 2015 Apr 25;16(1):191. doi: 10.1186/ s13063-015-0713-2.
15. Hamine S, Gerth-Guyette E, Faulx D, Green BB, Ginsburg AS. Impact of mHealth chronic disease management on treatment adherence and patient outcomes: a systematic review. J Med Internet Res. 2015 Feb 24;17(2):e52. doi: 10.2196/jmir.3951.

16. Blaya JA, Fraser HS, Holt B. E-health technologies show promise in developing countries. Health Aff (Millwood). 2010 Feb;29(2):244-51. doi: 10.1377/hlthaff.2009.0894.

17. Diez-Canseco F, Zavala-Loayza JA, Beratarrechea A, Kanter R, RamirezZea M, Rubinstein A, et al. Design and Multi-Country Validation of Text Messages for an mHealth Intervention for Primary Prevention of Progression to Hypertension in Latin America. JMIR Mhealth Uhealth. 2015 Feb 18;3(1):e19. doi: 10.2196/ mhealth.3874.

Correspondencia: Andrea Cecilia Rivas Nieto.

Dirección: Jr. Larco Herrera 1180 Dpto. 1201, Magdalena del Mar.

Teléfono: 961748116.

Correo electrónico: andrea.rivas@upch.pel andrisearch@gmail.com

\section{Consulte la versión electrónica de la Revista Peruana de Medicina Experimental y Salud Pública en} www.pubmed.gov

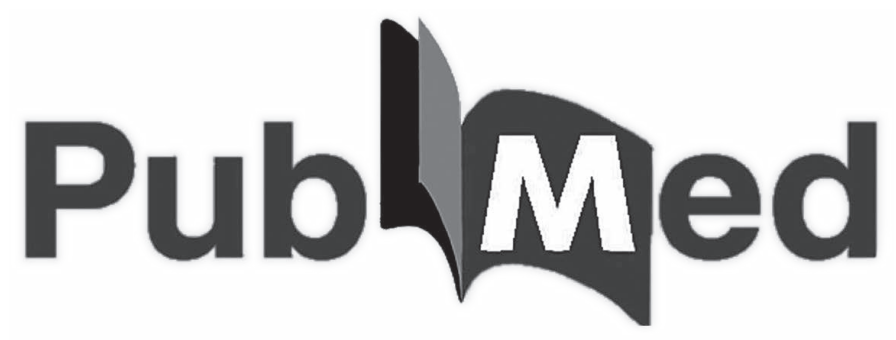

